Biostatistics (2012), ?, ?, pp. 1-27

doi:10.1093/biostatistics/bio000

\title{
Optimal sampling ratios in comparative diagnostic trials
}

\author{
Ting Dong \\ Department of Statistics, \\ George Mason University, Fairfax, VA 22030 \\ LIANSHENG TANG * \\ Department of Statistics, \\ George Mason University, Fairfax, VA 22030 \\ ltang1@gmu.edu \\ WILLIAM F. ROSENBERGER \\ Department of Statistics, \\ George Mason University, Fairfax, VA 22030
}




\section{SUMMARY}

Diagnostic trials evaluating a single marker or comparing two markers often employ an arbitrary sampling ratio between the case and the control groups. Such a ratio is not always an efficient choice when the goal is to maximize the power or to minimize the total required sample size. Instead, optimal sampling ratios, discussed by Janes and Pepe (2006), offer a better alternative for one-marker trials. In this paper we focus on comparative diagnostic trials which are frequently employed to compare two markers with continuous or ordinal results. We derive explicit expressions for the optimal sampling ratio based on a common variance structure shared by many existing summary statistics of the receiver operating characteristic (ROC) curve. Estimating the optimal ratio requires either pilot data or parametric model assumptions; however, pilot data are often unavailable at the planning stage of diagnostic trials. In the absence of pilot data, some distributions have to be assumed for carrying out the calculation. An optimal ratio from an incorrect distributional assumption may lead to an underpowered study. We propose a two-stage procedure to adaptively estimate the optimal ratio in comparative diagnostic trials without pilot data or assuming parametric distributions. We illustrate the properties of the proposed method through theoretical proofs and extensive simulation studies. We use an example in cancer diagnostic studies to illustrate the application of our method. We find that our method increases the power, or reduces the required overall sample size dramatically.

Keywords: AUC; Diagnostic accuracy; Internal pilot data; Two-stage design 


\section{INTRODUCTION}

Diagnostic trials estimate the diagnostic accuracy of a marker or compare the diagnostic accuracy of two markers. For example, in a diagnostic trial by Hendrick and others (2008), investigators compared the accuracy of digital mammography with screen-film mammography. Pepe and others (2001) refer to these trials as phrase III diagnostic trials. In these trials, the true disease status of subjects is known. To evaluate the diagnostic accuracy of a binary marker, sensitivity and specificity are used. Sensitivity is the probability of having a positive test result for a case subject. Specificity is the probability of having a negative test result for a control subject. The false positive rate (FPR) is 1 -specificity. For continuous markers, we obtain sensitivity and false positive rate (FPR) based on a threshold that distinguishes the test result as being positive or negative. A varying threshold allows a number of sensitivities and FPRs to be computed simultaneously. The receiver operating characteristic (ROC) curve is a plot of sensitivity versus FPR for all possible thresholds.

Typically the ratio between the number of cases versus the number of controls is fixed in advance. Most diagnostic trials apply an equal case-control ratio; for example, a lung cancer prevention trial recruited 71 prostate cancer cases and 71 age-matched controls without cancer (Etzioni and others, 2003). A diagnostic study in Hendrick and others (2008) compared the accuracy of digital mammography with screen-film mammography using equal numbers of breast cancer patients and controls. In a colorectal cancerscreening study, about the same number of colorectal cancer patients and non-cancer subjects were used to identify markers (Janes and others, 2005). The equal ratio, however, may not be optimal in maximizing the test power or minimizing the total required sample size. A procedure proposed by Janes and Pepe (2006) estimates the optimal ra- 
tio for evaluating a continuous marker. The ratio is optimal with regard to minimizing the variance, or maximizing the power for a fixed total required sample size. Equivalently, the optimal ratio minimizes the total required sample size with a fixed power. To the best of our knowledge, their method is the first attempt to identify the optimal sampling ratio in diagnostic trials. However, since the optimal ratio is derived using the first derivative of the ROC curve, their method cannot be used for ordinal data which often occur in medical imaging studies. More importantly, pilot data are required to estimate the optimal ratio. In the absence of pilot data, some distributions have to be assumed for carrying out the calculation. An optimal ratio from an incorrect distributional assumption may lead to an underpowered study. In addition, optimal ratios for comparative diagnostic trials are of interest to investigators, but have not been discussed in the literature.

In this paper we derive the optimal sampling ratio of cases to controls in comparative diagnostic trials. The proposed optimal ratio is based on a common variance structure shared among existing ROC summary statistics. Special cases of these statistics include the nonparametric area under the ROC curve (AUC) statistic proposed by DeLong and others (1988) and the weighted AUC statistic by Wieand and others (1989). These statistics have been applied in the sequential diagnostic trial design by Mazumdar and Liu (2003) and Liu and others (2008). The calculation of the optimal sampling ratio requires either parametric model assumptions or pilot data. When the parametric model is incorrectly specified, the resulting ratio may not give the optimal power or the minimal required sample size. It is desirable to re-calculate the optimal ratio when data become available during the trial. We propose a two-stage method to incorporate the idea of internal pilot data, reviewed in Proschan (2004). We assume a parametric model 
at the beginning of the trial to obtain the initial optimal ratio. This ratio is used to sample the cases and controls at the first stage. When sufficient observations are available, the optimal ratio is re-calculated at the second stage, and the numbers of cases and controls are adjusted accordingly. We show that although the optimal ratio is updated during a diagnostic trial, the analysis at the end of the trial can be carried out in the same fashion as in the traditional trial without affecting the nominal type I error rate.

The paper is organized as follows. In Section 2, we start with the optimal ratio for comparative diagnostic trials based on common ROC statistics. We then present the explicit expressions of the optimal ratios for comparing AUCs and for comparing weighted AUCs. In Section 3, we propose a two-stage procedure to adaptively estimate the optimal sampling ratio using the internal pilot data. We illustrate the power increase and the savings on the overall required sample size using the proposed method through a cancer example in Section 4. Section 5 investigates the small sample performance of the proposed procedure in maintaining the nominal type I error rate and increasing the power. Some discussion is presented in Section 6.

\section{OPTIMAL SAMPLING RATIO}

Suppose we have $N$ subjects with $m$ cases and $n$ controls. Each subject is measured by diagnostic test $\ell(\ell=1,2)$. We define the $i$ th case as $X_{\ell i}$, where $i=1, \ldots, m$, and the $j$ th control as $Y_{\ell j}$, where $j=1, \ldots, n$. The joint cumulative survival functions for cases are $\left(X_{1 i}, X_{2 i}\right) \sim S_{d}\left(x_{1}, x_{2}\right)$ and the joint cumulative survival functions for controls are $\left(Y_{1 j}, Y_{2 j}\right) \sim S_{\bar{d}}\left(y_{1}, y_{2}\right)$. Their marginal survival distributions are $X_{\ell i} \sim S_{d, \ell}(x)$ and $Y_{\ell j} \sim S_{\bar{d}, \ell}(y)$ respectively. For the threshold $c$ varying in $(-\infty,+\infty)$, the sensitivity is $S_{d, \ell}(c)=\operatorname{Pr}\left(X_{\ell i}>c\right)$, and the FPR is $S_{\bar{d}, \ell}(c)=\operatorname{Pr}\left(Y_{\ell j}>c\right)$. Subsequently, the ROC 
curve for test $\ell$ is defined as $R_{\ell}(u)=S_{d, \ell}\left(S_{\bar{d}, \ell}^{-1}(u)\right)$, where the FPR, $u$, falls within $[0,1]$.

Summary measures for a single ROC curve include the area under the ROC curve (AUC), the partial AUC (pAUC), and the weighted AUC (wAUC). The AUC gives the probability that a measurement randomly selected from the case group is greater than the measurement randomly selected from the control group (Bamber, 1975; Hanley and McNeil, 1982); that is, $\operatorname{Pr}(X>Y)=\int_{0}^{1} S_{d}\left\{S_{\bar{d}}^{-1}(u)\right\} d u$. The wAUC by Wieand and others (1989) is given by

$$
\Omega=\int_{0}^{1} S_{d}\left\{S_{\bar{d}}^{-1}(u)\right\} d W(u),
$$

where $W(u)$ is a probability measure. We let $W(u)$ be a point $u_{0}$, a FPR, to calculate the sensitivity of a test, or $W(u)=u$, where $u \in(0,1)$, to estimate the AUC. When $W(u)=\left(u-u_{0}\right) /\left(u_{1}-u_{0}\right)$, where $u \in\left(u_{0}, u_{1}\right),(2.1)$ gives the partial AUC.

The statistics for comparing markers might be parametric, e.g., the binormal model of Dorfman and Alf (1969), semiparametric (Zou and others, 1997; Tang and Zhou, 2009), or nonparametric (Mazumdar and Liu, 2003; DeLong and others, 1988; Hanley and McNeil, 1983; Wieand and others, 1989). Let $\theta$ be the parameter in the ROC comparison, and $\hat{\theta}$ be the estimator. Based on the variance expressions for these ROC statistics, we identify the following common structure for the variance of all these ROC statistics when the sample sizes get large:

$$
\operatorname{var}(\hat{\theta})=\frac{v_{x}}{m}+\frac{v_{y}}{n}
$$

where $v_{x}$ is the variance associated with measurements of case patients and $v_{y}$ is the variance related to control patients. In this paper we use the nonparametric statistics 
by DeLong and others (1988) and Wieand and others (1989). We present the variance expressions for these statistics in Section 2.1 and 2.2. One may refer to other aforementioned articles for the same variance structure of parametric and semiparametric ROC statistics.

Given the variance structure in (2.2), the total required sample size in a diagnostic trial can be minimized using an optimal sampling ratio when the variance is fixed. In other words, the power for comparing two markers can be maximized using this optimal sampling ratio. Suppose the total required sample size in the diagnostic trials is $N=$ $m+n$, the sampling ratio is $r=m / n$. Let the variance of $\hat{\theta}$ is a fixed constant, $a$. Since $m=r n=N r /(1+r)$, it follows that

$$
v_{x} / m+v_{y} / n=\frac{1+r}{N}\left(v_{x} / r+v_{y}\right)=a
$$

The total required sample size can then be expressed as

$$
N=\frac{1+r}{a}\left(v_{x} / r+v_{y}\right)
$$

To minimize $N$, we take first derivative with respect to $r$ and equate it to zero. We obtain the following equation:

$$
v_{y} / a-v_{x} / a r^{-2}=0
$$

By solving the equation above, the optimal sampling ratio is obtained as

$$
r^{*}=\sqrt{\frac{v_{x}}{v_{y}}} .
$$


The optimal sampling ratio is analogous to the Neyman allocation ratio for clinical trials which has been widely used to save the overall sample size for a fixed power. Interested readers can refer to Jennison and Turnbull (2000) and Rosenberger and Lachin (2002).

\subsection{Optimal sampling ratio for comparing two continuous markers}

The difference between two wAUCs, $\Delta=\Omega_{1}-\Omega_{2}$, is used in Wieand and others (1989) to compare the wAUCs for continuous data. Here the estimator $\hat{\Omega}_{\ell}$ of $\Omega_{\ell}$, for $\ell=1,2$, is obtained by substituting the empirical function estimators in (2.1). The

resulting $\Delta$-statistic is given by $\hat{\Delta}=\hat{\Omega}_{1}-\hat{\Omega}_{2}$. Let $w_{i}$ be $\int_{0}^{1}\left[S_{d, 1}\left(S_{\bar{d}, 1}^{-1}(u)\right)-I\left(X_{1 i} \leqslant\right.\right.$ $\left.\left.S_{\bar{d}, 1}^{-1}(u)\right)-S_{d, 2}\left(S_{\bar{d}, 2}^{-1}(u)\right)+I\left(X_{2 i} \leqslant S_{\bar{d}, 2}^{-1}(u)\right)\right] d W(u)$, and let $v_{j}$ be $\int_{0}^{1}\left\{R_{1}^{\prime}(u)\left[I\left(Y_{1 j} \leqslant\right.\right.\right.$ $\left.\left.\left.S_{\bar{d}, 1}^{-1}(u)\right)-u\right]-R_{2}^{\prime}(u)\left[I\left(Y_{2 j} \leqslant S_{\bar{d}, 2}^{-1}(u)\right)-u\right]\right\} d W(u)$, Tang and others (2008) further study the $\Delta$-statistic and show that for large sample sizes, $\hat{\Delta}$ is asymptotically equivalent to

$$
\frac{1}{m} \sum_{i=1}^{m} w_{i}+\frac{1}{n} \sum_{j=1}^{n} v_{j}+\left(\Omega_{1}-\Omega_{2}\right) .
$$

Since $w_{i}$ 's are i.i.d. random variables corresponding to measurements of case patients and $v_{j}$ 's are also i.i.d. random variables related to measurements of control subjects, (2.3) gives the optimal ratio for comparing the difference between wAUCs:

$$
r^{*}=\sqrt{\frac{\operatorname{var}\left(w_{i}\right)}{\operatorname{var}\left(v_{j}\right)}},
$$


where $\operatorname{var}\left(w_{i}\right)$ is given by the following expression:

$$
\begin{aligned}
\operatorname{var}\left(w_{i}\right)= & \sum_{\ell=1}^{2}\left(\int_{0}^{1} \int_{0}^{1} S_{d, \ell}\left\{S_{\bar{d}, \ell}^{-1}(s \wedge t)\right\} d W(s) d W(t)-\left[\int_{0}^{1} S_{d, \ell}\left\{S_{\bar{d}, \ell}^{-1}(s)\right\} d W(s)\right]^{2}\right) \\
& -2 \int_{0}^{1} \int_{0}^{1}\left[S_{d}\left\{S_{\bar{d}, 1}^{-1}(s), S_{\bar{d}, 2}^{-1}(t)\right\}-S_{d, 1}\left\{S_{\bar{d}, 1}^{-1}(s)\right\} S_{d, 2}\left\{S_{\bar{d}, 2}^{-1}(t)\right\}\right] d W(s) d W(t),
\end{aligned}
$$

and $\operatorname{var}\left(v_{j}\right)$ is given by the following expression:

$$
\begin{aligned}
\operatorname{var}\left(v_{j}\right)= & \sum_{\ell=1}^{2}\left[\int_{0}^{1} \int_{0}^{1} R_{\ell}^{\prime}(s) R_{\ell}^{\prime}(t)(s \wedge t) d W(s) d W(t)-\left\{\int_{0}^{1} r_{\ell}(s) s d W(s)\right\}^{2}\right] \\
& -2 \int_{0}^{1} \int_{0}^{1} R_{1}^{\prime}(s) R_{2}^{\prime}(t)\left[S_{\bar{d}}\left\{S_{\bar{d}, 1}^{-1}(s), S_{\bar{d}, 2}^{-1}(t)\right\}-s t\right] d W(s) d W(t),
\end{aligned}
$$

with the derivative of $R O C_{\ell}(u), R_{\ell}^{\prime}(u)=S_{d, \ell}^{\prime}\left\{S_{\bar{d}, \ell}^{-1}(u)\right\} / S_{\bar{d}, \ell}^{\prime}\left\{S_{\bar{d}, \ell}^{-1}(u)\right\}$.

Since $\hat{\Delta}$ compares AUCs, partial AUCs or sensitivities at a particular FPR, we discuss the optimal ratios for these special cases by specifying corresponding weight functions. When we let the weight function be $W(u)=u$, for $0<u<1, \hat{\Delta}$ compares the AUCs. The optimal ratio in (2.5) implies that the following ratio between the case and the control maximizes the power for comparing the AUCs:

$$
r_{A}^{*}=\sqrt{\frac{v_{x}^{A}}{v_{y}^{A}}},
$$

where $v_{x}^{A}$ and $v_{y}^{A}$ have the following expressions as shown in the Appendix:

$$
\begin{aligned}
v_{x}^{A}= & \sum_{\ell=1}^{2}\left(E\left[I\left(X_{\ell i}>Y_{\ell j}\right) I\left(X_{\ell i}>Y_{\ell l}\right)\right]-\left[E\left(I\left(X_{\ell i}>Y_{\ell j}\right)\right)\right]^{2}\right) \\
& -2\left(E\left[I\left(X_{1 i}>Y_{1 j}\right) I\left(X_{2 i}>Y_{2 l}\right)\right]-E\left[I\left(X_{1 i}>Y_{1 j}\right)\right] E\left[I\left(X_{2 i}>Y_{2 l}\right)\right]\right),
\end{aligned}
$$


and

$$
\begin{aligned}
v_{y}^{A}= & \sum_{\ell=1}^{2}\left(E\left[I\left(X_{\ell i}>Y_{\ell j}\right) I\left(X_{\ell k}>Y_{\ell j}\right)\right]-\left[E\left(I\left(X_{\ell i}>Y_{\ell j}\right)\right)\right]^{2}\right) \\
& -2\left(E\left[I\left(X_{1 i}>Y_{1 j}\right) I\left(X_{2 k}>Y_{2 j}\right)\right]-E\left[I\left(X_{1 i}>Y_{1 j}\right)\right] E\left[I\left(X_{2 k}>Y_{2 j}\right)\right]\right) .
\end{aligned}
$$

The optimal ratio for evaluating one marker, say marker 1, is simply

$$
\sqrt{\frac{E\left[I\left(X_{1 i}>Y_{1 j}\right) I\left(X_{1 i}>Y_{1 l}\right)\right]-\left[E\left(I\left(X_{1 i}>Y_{1 j}\right)\right)\right]^{2}}{E\left[I\left(X_{1 i}>Y_{1 j}\right) I\left(X_{1 k}>Y_{1 j}\right)\right]-\left[E\left(I\left(X_{1 i}>Y_{1 j}\right)\right)\right]^{2}}} .
$$

Janes and Pepe (2006) derive this ratio in terms of placement values as

$$
\sqrt{\frac{\operatorname{Var}\left(S_{\bar{d}, 1}\left(Y_{1 j}\right)\right)}{\operatorname{Var}\left(S_{d, 1}\left(X_{1 i}\right)\right)}}
$$

When $W(u)=I\left\{u=u_{0}\right\}$, where $0<u_{0}<1$, the $\hat{\Delta}$-statistic compares the sensitivities at the FPR $u_{0}$. The optimal ratio in (2.5) reduces to

$$
r_{s}^{*}=\sqrt{\frac{\left.\left.\sum_{\ell=1}^{2}\left\{R_{\ell}\left(u_{0}\right)\right\}-\left[R_{\ell}\left(u_{0}\right)\right\}\right]^{2}\right\}-2 A}{\sum_{\ell=1}^{2}\left\{R_{\ell}^{\prime}\left(u_{0}\right)^{2}-\left[R_{\ell}^{\prime}\left(u_{0}\right) u_{0}\right]^{2}\right\}-2 B}},
$$

where

$$
A=\operatorname{Pr}\left(X_{1 i}>G_{1}^{-1}\left(u_{0}\right), X_{2 i}>G_{2}^{-1}\left(u_{0}\right)\right)-R_{1}\left(u_{0}\right) R_{2}\left(u_{0}\right)
$$

and

$$
B=R_{1}^{\prime}\left(u_{0}\right) R_{2}^{\prime}\left(u_{0}\right)\left[\operatorname{Pr}\left(X_{1 i}>G_{1}^{-1}\left(u_{0}\right), X_{2 i}>G_{2}^{-1}\left(u_{0}\right)\right)-u_{0}^{2}\right] .
$$

The optimal ratio for evaluating marker 1 at the FPR $u_{0}$ is reduced to the ratio derived in Janes and Pepe (2006) as $\sqrt{R_{1}\left(u_{0}\right)\left(1-R_{1}\left(u_{0}\right)\right) /\left[u_{0}\left(1-u_{0}\right)\right]} / R_{1}^{\prime}\left(u_{0}\right)$. 


\subsection{Optimal sampling ratio for comparing two ordinal markers}

The variance of the $\hat{\Delta}$-statistic involves the first derivatives of the ROC curves. The optimal ratio in (2.5) cannot be readily applied to the ordinal data which often occur in radiology. In addition, the $\hat{\Delta}$-statistic does not allow for ties in marker observations. We thus consider the nonparametric statistic by DeLong and others (1988) to obtain the optimal ratio for comparing two ordinal markers which are usually two imaging modalities in radiology. DeLong's statistic estimates $P\left(X_{1 i}>Y_{1 j}\right)-P\left(X_{2 i}>Y_{2 j}\right)+$ $\left[P\left(X_{1 i}=Y_{1 j}\right)-P\left(X_{2 i}=Y_{2 j}\right)\right] / 2$, and is given as:

$$
\hat{\Delta}^{D}=\frac{1}{m n} \sum_{j=1}^{n} \sum_{i=1}^{m}\left[\psi\left(X_{1 i}, Y_{1 j}\right)-\psi\left(X_{2 i}, Y_{2 j}\right)\right]
$$

where $\psi\left(X_{\ell i}, Y_{\ell j}\right)=1$, for $Y_{\ell j}<X_{\ell i} ; 1 / 2$ for $Y_{\ell j}=X_{\ell i}$; and 0 for $Y_{\ell j}>X_{\ell i}$, for marker $\ell, \ell=1,2$. Let $\Omega_{\ell}^{A}$ be $P\left(X_{\ell i}>Y_{\ell j}\right)+P\left(X_{\ell i}=Y_{\ell j}\right) / 2$ for marker $\ell$, and $\hat{\Omega}_{\ell}^{A}$ be its estimator. DeLong and others (1988) show that the large sample variance of $\hat{\Delta}^{D}$ has the form of $\operatorname{var}\left(\hat{\Delta}^{D}\right)=v_{x}^{D} / m+v_{y}^{D} / n$, with

$$
\begin{gathered}
v_{x}^{D}=\frac{1}{m-1} \sum_{i=1}^{m}\left\{\left[\frac{1}{n} \sum_{j=1}^{n} \psi\left(X_{1 i}, Y_{1 j}\right)-\widehat{\Omega}_{1}^{A}\right]^{2}+\left[\frac{1}{n} \sum_{j=1}^{n} \psi\left(X_{2 i}, Y_{2 j}\right)-\widehat{\Omega}_{2}^{A}\right]^{2}\right. \\
\left.-2\left[\frac{1}{n} \sum_{j=1}^{n} \psi\left(X_{1 i}, Y_{1 j}\right)-\widehat{\Omega}_{1}^{A}\right]\left[\frac{1}{n} \sum_{j=1}^{n} \psi\left(X_{2 i}, Y_{2 j}\right)-\widehat{\Omega}_{2}^{A}\right]\right\}
\end{gathered}
$$


and

$$
\begin{gathered}
v_{y}^{D}=\frac{1}{n-1} \sum_{j=1}^{n}\left\{\left[\frac{1}{m} \sum_{i=1}^{m} \psi\left(X_{1 i}, Y_{1 j}\right)-\widehat{\Omega}_{1}^{A}\right]^{2}+\left[\frac{1}{m} \sum_{i=1}^{m} \psi\left(X_{2 i}, Y_{2 j}\right)-\widehat{\Omega}_{2}^{A}\right]^{2}\right. \\
\left.-2\left[\frac{1}{m} \sum_{i=1}^{m} \psi\left(X_{1 i}, Y_{1 j}\right)-\widehat{\Omega}_{1}^{A}\right]\left[\frac{1}{m} \sum_{i=1}^{m} \psi\left(X_{2 i}, Y_{2 j}\right)-\widehat{\Omega}_{2}^{A}\right]\right\} .
\end{gathered}
$$

Therefore, it follows from (2.3) that the ratio, $r_{D}^{*}=\sqrt{v_{x}^{D} / v_{y}^{D}}$, maximizes the power for comparing two ordinal markers.

\section{A TWO-STAGE PROCEDURE TO OBTAIN THE OPTIMAL RATIO}

One may assume a parametric model to obtain the variances and resulting optimal ratios derived in the preceding section. When a parametric model is correctly specified, the optimal ratio can be calculated from (2.3) for comparing ROC summary measures, and the sample size to obtain a specified power can be subsequently derived. However, if the parametric model is mis-specified, the calculated sample size may not give the appropriate power. We calculated the optimal ratios for comparing the AUCs or pAUCs from binormal and bi-exponential distributions. When comparing the AUCs, the optimal ratio is close to 1 for a wide range of the correlation parameter values for bivariate normal distributions. This implies that equal sampling for two groups yields the maximum power for a fixed total required sample size. However, the optimal ratio is around 1.5 for bi-exponential distributions, indicating that sampling 50\% more in cases than controls yields the maximum power to detect a difference between markers. When comparing the pAUCs, Figure 1 shows the optimal ratios for bivariate normal distributions. The optimal sampling ratio varies from 0.94 to 1.03 when correlation coefficients between 
two markers vary from -1 to 1 . Based on these two examples, the mis-specification of parametric models at the planning stage may lead to an incorrect optimal ratio.

Proschan (2004) introduces the concept of internal pilot data which often refers to accumulated data after a trial is carried out for a certain period of time. To correct for the model mis-specification at the beginning of the trial, we propose a two-stage procedure to use internal pilot data after some observations are available during the trial. Suppose the total required sample size $N$ is fixed. Without loss of generality, we use a two-sided test in the proposed procedure. The procedure is given in the following steps:

- Step 1: Specify a parametric model to obtain $v_{x, 0}$ and $v_{y, 0}$, and the resulting initial optimal ratio, $r_{0}^{*}=\sqrt{v_{x, 0} / v_{y, 0}}$.

- Step 2: Use the ratio $r_{0}^{*}$ together with $v_{x, 0}, v_{y, 0}$ in the following sample size formula to calculate initial sample sizes $m_{0}$ and $n_{0}$ with power $1-\beta$ and the significance level $\alpha$ :

$$
m_{0}=\frac{\left(z_{\alpha / 2}+z_{\beta}\right)^{2}\left(v_{x}+r_{0}^{*} v_{y}\right)}{\Delta_{1}^{2}}
$$

and $n_{0}=N-m_{0}$, where $\Delta_{1}$ is the difference between ROC summary measures under the alternative hypothesis.

- Step 3: After sufficient marker measurements are available on $m_{1}$ cases and $n_{1}$ controls at the first stage, the variance expressions of either the $\Delta$-statistic (Wieand and others, 1989) or DeLong's statistic (DeLong and others, 1988) are recalculated using available data. These variance estimators, $\hat{v}_{x, 1}$ and $\hat{v}_{y, 1}$, are applied in (2.3) to re-calculate the optimal ratio, $\hat{r}^{*}=\sqrt{\hat{v}_{x, 1} / \hat{v}_{y, 1}}$. 
- Step 4: Continue the trial by recruiting $M_{2}$ cases and $N_{2}$ controls, where $M_{2}$ and $N_{2}$ are given by

$$
M_{2}=\frac{N \hat{r}^{*}}{1+\hat{r}^{*}}-m_{1} \quad \text { and } \quad N_{2}=\frac{N}{1+\hat{r}^{*}}-n_{1} .
$$

It is showed in Proschan (2004) that using the internal pilot data for comparing population means in clinical trials maintains the nominal type I error rate. The reason is that the sample variance obtained at the end of the first stage does not give any information for the sample mean at the end of the trial. The same relationship between the estimated variance and the test statistic is also true for the $\Delta$-statistic or DeLong's statistic, as stated in Proposition 1. The proof is provided in the Appendix.

Proposition 1: At the first stage when $m_{1}$ and $n_{1}$ get large, the variance estimated at the first stage does not give any information for the $\Delta$-statistic or DeLong's statistic at the end.

Proposition 1 shows that estimating variances and the resulting optimal ratio using data from the first stage do not reveal information about the estimated difference between two ROC statistics obtained at the end of the second stage. Thus, although the optimal ratio is updated during the trial, the analysis at the end of the trial can be carried out in the same fashion as in the trial without updating the optimal ratio. This is important in maintaining the proper type I error rate.

\section{EXAMPLE}

In this section, we applied our method to a cancer diagnostic trial (Goddard and Hinberg, 1990). In this study 135 cancer patients and 218 non-cancer patients were recruited. A 
traditional biomarker, A, and newly developed diagnostic biomarkers were used to test blood samples from each subject. The unit of measurement was mmol of product per minute per millilitre, IU/mm. Measurements are highly skewed for all the methods. We compared a new biomarker D and the reference biomarker A to illustrate the power increment and the sample size savings by using the proposed procedure. We assumed a contrast of $\Delta_{1}=0.05$ between AUCs and the type I error rate 0.05 for power and sample size calculation based on a two-sided alternative. At the first stage, we accrued data on $m_{1}=60$ cancer and $n_{1}=60$ noncancer patients, and obtained the variance estimates, $\hat{v}_{x, 1}=0.082$ and $\hat{v}_{y, 1}=0.035$, which resulted in the optimal case-control ratio, $\hat{r}^{*}=1.53$, from (2.3). Let $N$ be the overall sample size, which is 353 by summing the numbers of cases and controls. Using this optimal ratio in the expression (3.2) in Step 4 of the proposed procedure, the numbers of the cases and controls to be recruited in the second stage were calculated to be 153 and 80, respectively. The power using the optimal ratio was then $50.9 \%$ using the following equation:

$$
1-\beta=\Phi\left(\Delta_{1} \sqrt{\frac{N \hat{r}^{*}}{\left(1+\hat{r}^{*}\right)\left(\hat{v}_{x, 1}+\hat{v}_{y, 1} \hat{r}^{*}\right)}}-z_{\alpha / 2}\right) .
$$

This power offers $7 \%$ increment over the power $43.8 \%$ calculated using the equation above by replacing $\hat{r}^{*}$ with the original case-control ratio of 0.62 . We also investigated the savings on the overall sample size by using the proposed procedure. Using the original power $43.8 \%$ with the estimated optimal ratio, $\hat{r}^{*}=1.53$, the overall sample size was calculated to be to 292 with 177 cancer patients and 115 noncancer patients. This offers savings of 61 patients over the original ratio. 


\section{Simulation StUdies}

In this section, we demonstrated the performance of our method for maximizing power when comparing summary statistics of diagnostic tests. We compared the proposed twostep procedure with the equal case-control ratio and a fixed case-control ratio under three parametric models. Three pairs of AUCs and pAUCs were specified in advance. We used DeLong's statistic for comparing the AUCs and the $\Delta$-statistic for comparing the pAUCs. We simulated 5000 observations from bivariate normal (BN), bivariate lognormal (LN) and bivariate exponential (BE) distributions, respectively. The bivariate normal models had the forms of $\left(X_{1}, X_{2}\right) \sim N\left\{\left(\mu_{1}, \mu_{2}\right), \Sigma\right\}$ and $\left(Y_{1}, Y_{2}\right) \sim N\{(0,0), \Sigma\}$, where in the $2 \times 2$ matrix $\Sigma$, the diagonal elements are 1's and off-diagonal elements are $\rho$. We chose $\rho=0.1$ and $\rho=0.25$ in our simulations. $\mu_{1}$ and $\mu_{2}$ were computed according to three pairs of AUCs, $(0.70,0.75),(0.75,0.80)$ and $(0.70,0.80)$, respectively. For comparing the pAUCs with the FPR in the range of $(0,0.6),\left(\mu_{1}, \mu_{2}\right)$ were used for three pairs of pAUCs, $(0.30,0.35),(0.35,0.40)$ and $(0.30,0.40)$, respectively. The bivariate lognormal models had the forms of $\exp \left(X_{1}, X_{2}\right)$ and $\exp \left(Y_{1}, Y_{2}\right)$ for cases and controls, respectively. They had the same values of $\left(\mu_{1}, \mu_{2}\right)$ for the AUCs and pAUCs as above. And then, according to the algorithm in Gumbel (1960), the bivariate exponential random variables take the form $H(x, y)=H_{1}(x) H_{2}(y)\left[1+4 \rho\left\{1-H_{1}(x)\right\}\left\{1-H_{2}(y)\right\}\right]$, where $\rho \in[-0.25,0.25]$. We set $\rho$ be 0.1 and 0.25 here. The marginal survival functions for cases and controls were $\exp \left(-\beta_{\ell 1} x\right)$ and $\exp \left(-\beta_{\ell 2} y\right)$, so we could generate data from these two distributions respectively. In the simulation, we set $\beta_{11}=1$ and $\beta_{21}=1$. $\beta_{12}$ and $\beta_{22}$ were computed according to the AUC or pAUC values. For the pairs of AUCs $(0.70,0.75),(0.75,0.80)$, and $(0.70,0.80)$, the corresponding $\left(\beta_{12}, \beta_{22}\right)$ values were $(2.333,3.003),(3.003,4.000)$ and $(2.333,4.000)$. For the pairs of pAUCs 
$(0.30,0.35),(0.35,0.40)$ and $(0.30,0.40)$, the $\left(\beta_{12}, \beta_{22}\right)$ values were $(1.8957,2.5094)$, $(2.5094,3.3887)$ and $(1.8957,3.3887)$, respectively.

In our simulation, we first assumed that our samples were from bivariate normal distributions, then used equation (3.1) to calculate the initial total required sample size. With the type I error rate 0.05 and power $80 \%$, the initial total required sample sizes were $N=1421,1200$, or 326 to detect the difference of three pairs of AUCs of $(0.70,0.75)$, $(0.75,0.80)$ and $(0.70,0.80)$, respectively, with $\rho=0.1$. When $\rho=0.25$, the total required sample sizes, $N=1207,1025$, or 278 , were needed to detect the difference in these pairs. For comparing the pAUCs, the initial total required sample sizes were $N=1067,979$, and 251, for for $\rho=0.1$, and $N=915,842$ and 216 for $\rho=0.25$. There were three different sampling ratios: 1) the proposed two-stage optimal ratio; 2) fixed sample ratio of $0.5 ; 3$ ) equal sampling ratio. To implement the proposed method, we defined the number of available observations at the first stage, $m_{1}=n_{1}=N / 4$. By substituting nonparametric variance estimates $\hat{v}_{x, 1}$ and $\hat{v}_{y, 1}$, the resulting optimal ratio was estimated by $\hat{r}^{*}=\sqrt{\hat{v}_{x, 1} / \hat{v}_{y, 1}}$, and $M_{2}$ and $N_{2}$ were calculated using (3.2). We then generated $M_{2}$ new observations for cases and $N_{2}$ observations for controls. Subsequently, the null hypothesis of equal AUCs or pAUCs was rejected in favor of the alternative if the $Z$-statistic calculated using all simulated data was greater than or equal to $z_{0.025}$. The simulated power was then calculated as the percent of times out of 5000 that the null hypothesis was rejected. The simulated powers for all simulation settings are present in Table 1.

Table 1 illustrates that larger correlations resulted in higher rejection rates. The sampling ratio is another factor impacting the power when the alternative hypothesis is true. For different underlying distributions, the proposed two-stage method has higher powers 
than the fixed ratios in most of the settings.

We also evaluated the performance of the two-step procedure to see whether the procedure maintains the nominal type I error rate. We used the total required sample sizes, $N=200,400$, or 500. The parametric distributions and three different sampling ratios used in the previous simulation were considered. We assumed equal AUCs or pAUCs with the AUCs being $(0.70,0.75,0.80)$, and the pAUCs being $(0.30,0.35,0.40)$. The nominal type I error rate was 0.05 in our simulation. The simulated type I error rates are shown in Table 2. All these rates are close to the nominal level when the sample size goes to 500 .

Variability in the estimators, $\hat{v}_{x, 1}$ and $\hat{v}_{y, 1}$, is associated with the initial sample sizes at the first stage. Such variability affects the calculation of the optimal sampling ratio, which may in turn have an impact on the power in the proposed procedure. We conducted another simulation study to investigate the impact of the initial sample size selection. We used the total required sample size of 400 , and set the initial sample sizes of cases and controls to be $m_{0}=n_{0}=50,60,80$, or 100. Observations were simulated from the binormal distributions with the difference of 0.05 between two AUCs. In each simulation, the variance estimators for calculating the optimal ratio were estimated at the first stage from three scenarios, namely, 1) a single set of $m_{0}$ cases and $n_{0}$ controls, 2) averaging variance estimates of 10 sets of $m_{0}$ cases and $n_{0}$ controls, and 3 ) averaging variance estimates of 100 sets of $m_{0}$ cases and $n_{0}$ controls. Results based on 1000 replications for each setting are listed in Table 3. It indicates some variations in power for the first scenario. When more datasets are involved in the calculation, power becomes more stable regardless of the initial sample sizes. More importantly, Table 3 shows that the initial sample size selection had little impact on the final power. 


\section{CONCLUSION}

The optimal sampling ratio in diagnostic trials can maximize the test power or minimize the overall sample size. The optimal sampling ratio discussed in this paper is analogous to the optimal allocation ratio in assigning patient treatments in clinical trials. The optimal allocation ratio has been used in clinical trials for decades, but the importance of the optimal ratio in diagnostic trials has not been widely recognized. Implementation requires the calculation of complicated variances of frequently used ROC statistics. This paper discusses a common variance structure for ROC statistics, and thereby introduces optimal sampling ratios in comparative diagnostic trials based on these statistics. Two popular nonparametric ROC statistics are used to illustrate the explicit forms of the optimal ratios because their variance expressions can be written as the sum of separate terms; one relates to the cases, and the other relates to the controls. The same variance structure is shared by many existing parametric and semiparametric ROC statistics. This implies that the optimal ratio form derived in (2.3) is also applicable to these existing statistics.

When marker results follow normal distributions, the optimal sampling ratio is close to 1 for many parameter settings. Then sampling the same number of cases and controls can potentially achieve the maximal power for a fixed total required sample size. When the marker results follow exponential distributions, the sampling ratio is close to 1.5 . We need to sample more cases than controls to gain power or reduce the overall sample size. If preliminary studies are available before carrying out a comparative diagnostic trial, the variance can be estimated using pilot data to obtain the optimal ratio for comparing specified ROC summary measures. The ratio can then be used to recruit patients in the trial, and re-calculating the ratio may not be necessary during the trial. However, 
when medical practitioners do not have preliminary data for the markers and are not certain about the distributions of the marker results, the distribution assumption used for obtaining the optimal ratio may be far from the true underlying distributions for the marker results. This may result in less power or larger overall sample sizes than using the true optimal ratio. The proposed two-stage procedure is then particularly useful to ensure that the optimal ratio can be re-calculated using using internal pilot data during the trial. The proposed procedure performed well in a large scale simulation study. We also demonstrated that the proposed procedure maintains the nominal type I error rate in the simulation. We used an example in cancer diagnostic studies to illustrate the application of our method on maximizing the test power and saving overall sample sizes. The results indicated that compared with the original sampling ratio, using the proposed two-stage procedure for a fixed overall sample size increased the test power. Alternatively, for the fixed test power, the proposed procedure reduced the overall sample size by nearly $25 \%$.

It is sometimes desired to minimize the total cost in a diagnostic trial with a limited budget. High cost may be associated with diagnostic trials considering using a gold standard test to identify the subjects and using markers to diagnosing them. This is particularly true in medical imaging diagnostic trials when expensive medical imaging devices costing hundreds of dollars for a single session of scans are involved. A case may cost more than a control because of higher expenses associated with providing necessary medical care when classifying and diagnosing them. We may consider $c_{1}$ and $c_{2}$ as costs related to a case and a control, respectively. Usually, $c_{1}$ and $c_{2}$ can be determined by medical experts before conducting a trial. Then similar to the derivation in Section 2, the optimal sampling ratio for minimizing the total cost is given by $r_{c}^{*}=\sqrt{c_{2} v_{x} / c_{1} v_{y}}$ for a fixed power. This ratio reduces to the one derived in (2.3) when $c_{1}=c_{2}$. An interesting 
future research topic is to investigate the optimal ratio when the costs are related to the true AUC parameters.

\section{REFERENCES}

BAMBER, D. (1975). The area above the ordinal dominance graph and the area below the receive operating characteristic graph. Journal of Mathematical Psychology 12, 387-415.

Delong, E. R., Delong, D. M. and Clarke-Pearson, D. L. (1988). Comparing the areas under two or more correlated receiver operating characteristic curves: A nonparametric approach. Biometrics 44, 837-845.

Dorfman, D. D. AND Alf, E. (1969). Maximum likelihood estimation of parameters of signal detection theory and determination of confidence intervals-rating method data. Journal of Mathematical Psychology 6, 487-496.

Etzioni, R., Kooperberg, C., Pepe, M., Smith, R. And Gann, P. H. (2003). Combining biomarkers to detect disease with application to prostate cancer. Biostatistics 4, 523-538.

Goddard, M. J. And Hinberg, I. (1990). Receiver operator characteristic (roc) curves and non-normal data: An empirical study. Statistics in Medicine 9, 325-337.

HANLEY, J.A. AND MCNEIL, B.J. (1983). A method of comparing the areas under (ROC) curves derived from same cases. Radiology 148, 839-843.

HANLEY, J. A. AND MCNEIL, B. J. (1982). The meaning and use of the area under a receiver operating characteristic (roc) curve. Radiology 143, 29-36.

Hendrick, R. E., Cole, E. B., Pisano, E. D., Acharyya, S., Marques, H., Cohen, M. A., Jong, R. A., Mawdsley, G. E., Kanal, K. M., D’Orsi, C. J., Rebner, M. and others. (2008). Accuracy of soft-copy digital mammography versus that of screen-film mammography according to digital manufacturer: Acrin dmist retrospective multireader study. Radiology 247, 38-48.

Janes, H. And PePE, M. (2006). The optimal ratio of cases to controls in a case-control for estimating the classification accuracy of a biomarker. Biostatistics 7, 456-468.

Janes, H., Pepe, M. S., Kooperberg, C. And Newcomb, P. (2005). Identifying target populations for screening or not screening using logic regression. Statistics in Medicine 24, 1321-1338.

Jennison, C. And Turnbull, B. W. (2000). Group Sequential Methods with Applications to Clinical Trials. New 
York: Chapman and Hall.

LiU, Aiyi, Wu, Chengqing And Schisterman, Enrique F. (2008). Nonparametric sequential evaluation of diagnostic biomarkers. Statistics in Medicine 27(10), 1667-1678.

MAZumdar, MAdHU AND LiU, AiYI. (2003). Group sequential design for comparative diagnostic accuracy studies. Statistics in Medicine 22(5), 727-739.

Pepe, M. S., Etzioni, R., Feng, Z., Potter, J. D., M., Thompson., Thornquist, M., Winget, M. And YASUI, Y. (2001). Phases of biomarker development for early detection of cancer. Journal of the National Cancer Institute 93, 105461.

Proschan, M. (2004). Two-stage sample size re-estimation based on nuisance parameter a review. Journal of Biopharmaceutical Statistics 15, 559-574.

Rosenberger, W. F. And Lachin, J. M. (2002). Rondomization in Clinical Trials Theory and Practice. New York: John Wiley.

TANG, L., EMERSON, S. S. AND ZHOU, X. (2008). Nonparametric and semiparametric group sequential methods for comparing accuracy of diagnostic tests. Biometrics 64, 1137-1145.

TANG, Liansheng And Zhou, Xiao HuA. (2009). Semiparametric inferential procedures for comparing multivariate ROC curves with interaction terms. Statistica Sinica 19, 1137-1145.

Wieand, S., Gail, M. H., James, B. R. And James, K. L. (1989). A family of non-parametric statistics for comparing diagnostic markers with paired or unpaired data. Biometrika 76, 585-592.

ZOU, KH, HALl, WJ AND SHAPIRO, DE. (1997, OCT 15). Smooth non-parametric receiver operating characteristic (ROC) curves for continuous diagnostic tests. STATISTICS IN MEDICINE 16(19), 2143-2156.

\section{APPENDIX}

Appendix: variance derivation and proof of Proposition 1

Derivation of $v_{x}^{A}$ and $v_{y}^{A}$

We can show that

$$
\int_{0}^{1} \int_{0}^{1}\left[S_{d}\left\{S_{\bar{d}, 1}^{-1}(s), S_{\bar{d}, 2}^{-1}(t)\right\}\right] d s d t
$$


can be expressed as

$$
\int_{-\infty}^{\infty} \int_{-\infty}^{\infty} S_{d}\left(y_{1}, y_{2}\right) d S_{\bar{d}, 1}\left(y_{1}\right) d S_{\bar{d}, 2}\left(y_{2}\right)
$$

Let $S_{\bar{d}, 1}^{-1}(s)=y_{1}$ and $S_{\bar{d}, 2}^{-1}(t)=y_{2}$, then, we have

$$
\int_{0}^{1} \int_{0}^{1}\left[S_{d}\left\{S_{\bar{d}, 1}^{-1}(s), S_{\bar{d}, 2}^{-1}(t)\right\}\right] d s d t=E\left[I\left(X_{1 i}>Y_{1 j}\right) I\left(X_{2 i}>Y_{2 l}\right)\right]
$$

Similarly, $v_{y}$ becomes

$$
\begin{aligned}
v_{y}= & \sum_{\ell=1}^{2}\left[\int_{0}^{1} \int_{0}^{1} r_{\ell}(s) r_{\ell}(t)(s \wedge t) d s d t-\left\{\int_{0}^{1} r_{\ell}(s) s d s\right\}^{2}\right] \\
& -2 \int_{0}^{1} \int_{0}^{1} r_{1}(s) r_{2}(t)\left[S_{\bar{d}}\left\{S_{\bar{d}, 1}^{-1}(s), S_{\bar{d}, 2}^{-1}(t)\right\}-s t\right] d s d t
\end{aligned}
$$

It follows that

$$
\begin{gathered}
\int_{0}^{1} \int_{0}^{1} r_{1}(s) r_{2}(t) S_{\bar{d}}\left\{S_{\bar{d}, 1}^{-1}(s), S_{\bar{d}, 2}^{-1}(t)\right\} d s d t \\
=\int_{0}^{1} \int_{0}^{1} \frac{S_{d, 1}^{\prime}\left\{S_{\bar{d}, 1}^{-1}(s)\right\}}{S_{\bar{d}, 1}^{\prime}\left\{S_{\bar{d}, 1}^{-1}(s)\right\}} \frac{S_{d, 2}^{\prime}\left\{S_{\bar{d}, 2}^{-1}(t)\right\}}{S_{\bar{d}, 2}^{\prime}\left\{S_{\bar{d}, 2}^{-1}(t)\right\}} S_{\bar{d}}\left\{S_{\bar{d}, 1}^{-1}(s), S_{\bar{d}, 2}^{-1}(t)\right\} d s d t
\end{gathered}
$$

Let $S_{\bar{d}, 1}^{-1}(s)=y_{1}$ and $S_{\bar{d}, 2}^{-1}(t)=y_{2}$, then it follows that

$$
\begin{aligned}
& \left.\iint S_{d, 1}^{\prime}\left\{y_{1}\right\} S_{d, 2}^{\prime}\left\{y_{2}\right\} S_{\bar{d}}\left(y_{1}, y_{2}\right)\right) d y_{1} d y_{2} \\
= & E\left[I\left(X_{1 i}<Y_{1 j}\right) I\left(X_{2 k}<Y_{2 j}\right)\right] \\
= & E\left[\left(1-I\left(X_{1 i}>Y_{1 j}\right)\right)\left(1-I\left(X_{2 k}>Y_{2 j}\right)\right)\right] \\
= & 1-E\left(I\left(X_{1 i}>Y_{1 j}\right)\right)-E\left(I\left(X_{2 k}>Y_{2 j}\right)\right)+E\left[I\left(X_{1 i}>Y_{1 j}\right) I\left(X_{2 k}>Y_{2 j}\right)\right] .
\end{aligned}
$$


Because

$$
\int_{0}^{1} \int_{0}^{1} r_{1}(s) r_{2}(t) s t d s d t
$$

can also be written as

$$
1-\operatorname{Pr}\left(X_{1 i}>Y_{1 j}\right)-\operatorname{Pr}\left(X_{2 k}>Y_{2 j}\right)+E\left[I\left(X_{1 i}>Y_{1 j}\right)\right] E\left[I\left(X_{2 k}>Y_{2 j}\right)\right]
$$

the expressions for $v_{x}$ and $v_{y}$ are simplified as follows:

$$
\begin{aligned}
v_{x}^{A}= & \sum_{\ell=1}^{2}\left(E\left[I\left(X_{\ell i}>Y_{\ell j}\right) I\left(X_{\ell i}>Y_{\ell l}\right)\right]-\left[E\left(I\left(X_{\ell i}>Y_{\ell j}\right)\right)\right]^{2}\right) \\
& -2\left(E\left[I\left(X_{1 i}>Y_{1 j}\right) I\left(X_{2 i}>Y_{2 l}\right)\right]-E\left[I\left(X_{1 i}>Y_{1 j}\right)\right] E\left[I\left(X_{2 i}>Y_{2 l}\right)\right]\right)
\end{aligned}
$$

and

$$
\begin{aligned}
v_{y}^{A}= & \sum_{\ell=1}^{2}\left(E\left[I\left(X_{\ell i}>Y_{\ell j}\right) I\left(X_{\ell k}>Y_{\ell j}\right)\right]-\left[E\left(I\left(X_{\ell i}>Y_{\ell j}\right)\right)\right]^{2}\right) \\
& -2\left(E\left[I\left(X_{1 i}>Y_{1 j}\right) I\left(X_{2 k}>Y_{2 j}\right)\right]-E\left[I\left(X_{1 i}>Y_{1 j}\right)\right] E\left[I\left(X_{2 k}>Y_{2 j}\right)\right]\right) .
\end{aligned}
$$

\section{Proof of Proposition 1}

We first prove that the proposition is true for the $\Delta$-statistic. Similar arguments can then be used for the Delong's statistic. Let $\bar{w}=\frac{\sum_{i=1}^{m_{1}} w_{i}}{m_{1}}$. We see that in (4), wis are i.i.d. random variables independent of i.i.d. random variables $v_{j}$ 's. It then follows that

$$
\operatorname{cov}\left(\frac{\sum_{i=1}^{m_{1}+m_{2}} w_{i}}{m_{1}+m_{2}}, w_{i}-\bar{w}\right)=\operatorname{cov}\left(\frac{\sum_{i=1}^{m_{1}+m_{2}} w_{i}}{m_{1}+m_{2}}, w_{i}\right)-\operatorname{cov}\left(\frac{\sum_{i=1}^{m_{1}+m_{2}} w_{i}}{m_{1}+m_{2}}, \bar{w}\right)
$$


equals 0 , and

$$
\begin{aligned}
& \bar{w}=\int_{0}^{1} S_{d, 1}\left(S_{\bar{d}, 1}^{-1}(u)\right) d u-\frac{1}{m_{1}} \sum_{i=1}^{m_{1}} \int_{0}^{1} I\left(X_{1 i} \leqslant S_{\bar{d}, 1}^{-1}(u)\right) d u \\
& +\frac{1}{m_{1}} \sum_{i=1}^{m_{1}} \int_{0}^{1} I\left(X_{2 i} \leqslant S_{\bar{d}, 2}^{-1}(u)\right) d u-\int_{0}^{1} S_{d, 2}\left(S_{\bar{d}, 2}^{-1}(u)\right) d u .
\end{aligned}
$$

We then get

$$
\begin{gathered}
\left(w_{i}-\bar{w}\right)=\frac{1}{m_{1}} \sum_{i=1}^{m_{1}} \int_{0}^{1} I\left(X_{1 i} \leqslant S_{\bar{d}, 1}^{-1}(u)\right) d u-\int_{0}^{1} I\left(X_{1 i} \leqslant S_{\bar{d}, 1}^{-1}(u)\right) d u \\
+\int_{0}^{1} I\left(X_{2 i} \leqslant S_{\bar{d}, 2}^{-1}(u)\right) d u-\frac{1}{m_{1}} \sum_{i=1}^{m_{1}} \int_{0}^{1} I\left(X_{2 i} \leqslant S_{\bar{d}, 2}^{-1}(u)\right) d u .
\end{gathered}
$$

Therefore,

$$
\frac{\sum_{i=1}^{m_{1}}\left(w_{i}-\bar{w}\right)^{2}}{m_{1}-1} \approx \hat{v}_{x_{1}} / m_{1}
$$

which indicates that for large sample sizes, $\left(\sum_{i=1}^{m_{1}+m_{2}} w_{i}\right) /\left(m_{1}+m_{2}\right)$ is independent of $\hat{v}_{x, 1} / m_{1}$. Similarly, we get that for large sample sizes, $\left(\sum_{j=1}^{n_{1}+n_{2}} v_{j}\right) /\left(n_{1}+n_{2}\right)$ is independent of $\hat{v}_{y, 1} / n_{1}$. 


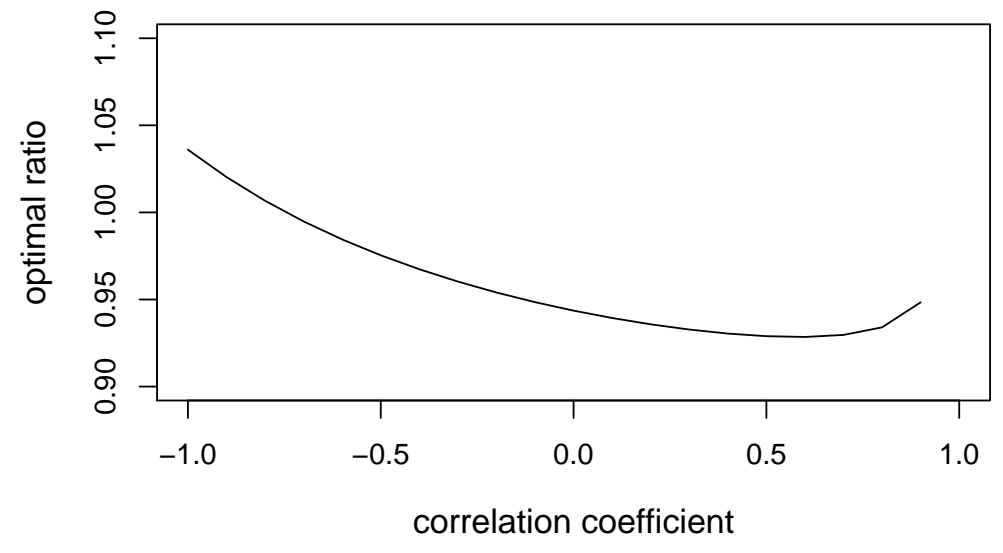

Fig. 1. Optimal sampling ratio for comparing pAUCs. The observations are from two bivariate normal distributions.

The FPR is between 0 and 0.6 . 
Table 1. Simulated power (in \%) for comparing AUCs or pAUCs

\begin{tabular}{|c|c|c|c|c|c|c|c|c|}
\hline \multicolumn{9}{|c|}{ Comparing AUCs using the DeLong's method } \\
\hline \multirow[b]{2}{*}{$\rho$} & & & \multicolumn{4}{|c|}{ Two-Stage } & $r=1$ & $r=0.5$ \\
\hline & & $\Omega_{1}^{A} \backslash \Omega_{2}^{A}$ & 0.70 & $A R$ & 0.75 & $A R$ & 0.700 .75 & 0.700 .75 \\
\hline \multirow{6}{*}{0.1} & $\overline{B N}$ & 0.75 & 79.3 & 1.001 & - & - & 79.7 & 74.6 \\
\hline & & 0.80 & 81.0 & 1.003 & 79.1 & 1.002 & 78.880 .5 & 75.374 .8 \\
\hline & $L N$ & 0.75 & 80.5 & 1.001 & - & - & 80.2 & 75.6 \\
\hline & & 0.80 & 80.3 & 1.003 & 79.4 & 1.000 & 79.880 .6 & 75.375 .2 \\
\hline & $B E$ & 0.75 & 81.0 & 1.340 & - & - & 80.4 & 71.2 \\
\hline & & 0.80 & 81.6 & 1.467 & 81.8 & 1.551 & 80.080 .4 & 70.069 .9 \\
\hline \multirow{7}{*}{0.25} & & $\Omega_{1}^{A} \backslash \Omega_{2}^{A}$ & 0.70 & $\overline{A R}$ & 0.75 & $\overline{A R}$ & 0.700 .75 & 0.700 .75 \\
\hline & $\overline{B N}$ & 0.75 & 79.8 & 1.002 & - & - & 80.0 & 74.9 \\
\hline & & 0.80 & 80.2 & 1.007 & 80.3 & 1.004 & 79.180 .2 & 75.174 .5 \\
\hline & $L N$ & 0.75 & 79.8 & 1.002 & - & - & 79.8 & 75.3 \\
\hline & & 0.80 & 80.2 & 1.005 & 79.8 & 1.003 & 79.779 .6 & 74.975 .3 \\
\hline & $B E$ & 0.75 & 83.7 & 1.412 & - & - & 82.6 & 74.2 \\
\hline & & 0.80 & 83.6 & 1.482 & 83.5 & 1.579 & $82.8 \quad 81.0$ & 72.571 .4 \\
\hline
\end{tabular}

Comparing pAUCs using the $\Delta$-statistic

\begin{tabular}{|c|c|c|c|c|c|c|c|c|c|}
\hline \multirow[b]{2}{*}{$\rho$} & \multirow{2}{*}{\multicolumn{2}{|c|}{$\Omega_{1}^{P A} \backslash \Omega_{2}^{P A}$}} & \multicolumn{4}{|c|}{ Two-Stage } & \multicolumn{2}{|c|}{$r=1$} & $r=0.5$ \\
\hline & & & 0.30 & $A R$ & 0.35 & $A R$ & 0.30 & 0.35 & 0.300 .35 \\
\hline \multirow{6}{*}{0.1} & $B N$ & 0.35 & 79.2 & 0.952 & - & - & 78.7 & & 73.9 \\
\hline & & 0.40 & 79.8 & 1.008 & 79.3 & 0.954 & 80.6 & 79.2 & 75.175 .0 \\
\hline & $L N$ & 0.35 & 79.0 & 0.953 & - & - & 78.9 & - & 74.8 \\
\hline & & 0.40 & 80.4 & 1.003 & 79.3 & 0.954 & 81.0 & 80.6 & 75.874 .8 \\
\hline & $B E$ & 0.35 & 84.6 & 1.249 & - & - & 84.0 & - & 76.6 \\
\hline & & 0.40 & 84.9 & 1.389 & 83.6 & 1.386 & 84.6 & 83.9 & 76.674 .4 \\
\hline \multirow{7}{*}{0.25} & & $\Omega_{1}^{P A} \backslash \Omega_{2}^{P A}$ & 0.30 & $A R$ & 0.35 & $A R$ & 0.30 & 0.35 & $\begin{array}{lll}0.30 & 0.35 \\
\end{array}$ \\
\hline & $B N$ & 0.35 & 78.7 & 0.947 & - & - & 78.0 & & 75.4 \\
\hline & & 0.40 & 80.6 & 1.014 & 78.7 & 0.952 & 80.5 & 79.8 & 76.075 .2 \\
\hline & $L N$ & 0.35 & 79.2 & 0.949 & - & - & 79.0 & - & 74.6 \\
\hline & & 0.40 & 80.9 & 1.013 & 79.3 & 0.950 & 80.7 & 79.2 & 76.675 .3 \\
\hline & $B E$ & 0.35 & 86.9 & 1.219 & - & - & 87.1 & - & 80.0 \\
\hline & & 0.40 & 86.8 & 1.385 & 84.5 & 1.365 & 86.6 & 84.0 & 78.676 .9 \\
\hline
\end{tabular}

AR - the average ratio, $\mathrm{BN}$ - bivariate normal, LN - bivariate lognormal, BE - bivariate exponential, $\Omega_{1}^{A}$ - the AUC for marker $1, \Omega_{2}^{A}$ - the AUC for marker $2, \Omega_{1}^{P A}$ - the pAUC for marker $1, \Omega_{2}^{P A}$ - the pAUC for marker $2, \rho$ - the correlation coefficient of two markers. 
Table 2. Type I error rates (in \%) for comparing the AUCs or pAUCs

\begin{tabular}{ccc|c|ccccccc}
\hline & \multicolumn{4}{c}{ Comparing the AUCs } & \multicolumn{3}{c}{ Comparing the pAUCs } \\
\hline$\rho$ & & AUCs & N=200 & 400 & 500 & pAUCs & N=200 & 400 & 500 \\
\hline \multirow{4}{*}{ BN } & 0.70 & 4.5 & 5.8 & 5.0 & 0.30 & 5.6 & 5.9 & 4.6 \\
& 0.75 & 5.4 & 5.8 & 4.0 & 0.35 & 6.8 & 5.8 & 5.0 \\
& & 0.80 & 5.3 & 5.1 & 5.2 & 0.40 & 6.7 & 5.5 & 6.1 \\
LN & 0.70 & 5.7 & 5.3 & 5.0 & 0.30 & 6.8 & 5.9 & 4.6 \\
& 0.75 & 5.4 & 6.5 & 4.3 & 0.35 & 6.8 & 5.8 & 5.0 \\
& 0.80 & 5.3 & 4.4 & 5.9 & 0.40 & 6.7 & 5.5 & 6.1 \\
& BE & 0.70 & 5.4 & 5.1 & 4.4 & 0.30 & 6.2 & 5.4 & 5.5 \\
& 0.75 & 5.3 & 6.6 & 4.0 & 0.35 & 7.7 & 7.4 & 5.6 \\
& 0.80 & 5.2 & 5.1 & 4.3 & 0.40 & 7.5 & 7.3 & 5.0 \\
\hline \multirow{4}{*}{ BN } & 0.70 & 4.9 & 4.8 & 4.7 & 0.30 & 5.9 & 5.3 & 5.4 \\
& 0.75 & 5.9 & 5.4 & 5.9 & 0.35 & 5.2 & 5.3 & 5.2 \\
& 0.80 & 5.5 & 6.1 & 5.6 & 0.40 & 5.6 & 5.8 & 5.1 \\
0.25 LN & 0.70 & 5.2 & 5.3 & 5.5 & 0.30 & 6.0 & 5.3 & 5.4 \\
& 0.75 & 5.9 & 5.4 & 5.9 & 0.35 & 5.2 & 5.3 & 5.2 \\
& 0.80 & 5.8 & 3.9 & 4.3 & 0.40 & 6.7 & 5.8 & 5.4 \\
BE & 0.70 & 4.2 & 5.0 & 3.9 & 0.30 & 5.0 & 5.6 & 4.8 \\
& 0.75 & 5.3 & 5.7 & 4.4 & 0.35 & 5.7 & 7.0 & 6.4 \\
& 0.80 & 5.2 & 5.1 & 4.3 & 0.40 & 6.8 & 6.8 & 6.4 \\
\hline
\end{tabular}

$\mathrm{BN}$ - bivariate normal, $\mathrm{LN}$ - bivariate lognormal, BE - bivariate exponential, $N$ - the total required sample size, $\rho$ - the correlation coefficient of two markers

Table 3. Power comparison

\begin{tabular}{|c|c|llll|}
\hline & & \multicolumn{4}{|c|}{$m_{0}\left(n_{0}\right)$} \\
\hline$K$ & & 50 & 60 & 80 & 100 \\
\hline 1 & Ratio & 1.02 & 1.01 & 0.87 & 1.07 \\
& Power (\%) & 31.9 & 26.6 & 32.6 & 33.7 \\
\hline 10 & Ratio & 1.03 & 0.94 & 1.03 & 1.05 \\
& Power (\%) & 29.9 & 31.3 & 31.9 & 32.5 \\
\hline \multirow{2}{*}{100} & Ratio & 1.03 & 1.02 & 1.00 & 1.03 \\
& Power (\%) & 31.1 & 30.8 & 31.1 & 31.4 \\
\hline
\end{tabular}

$K$ - the number of datasets simulated to estimate variances. 
Power size with various $\rho_{A}$

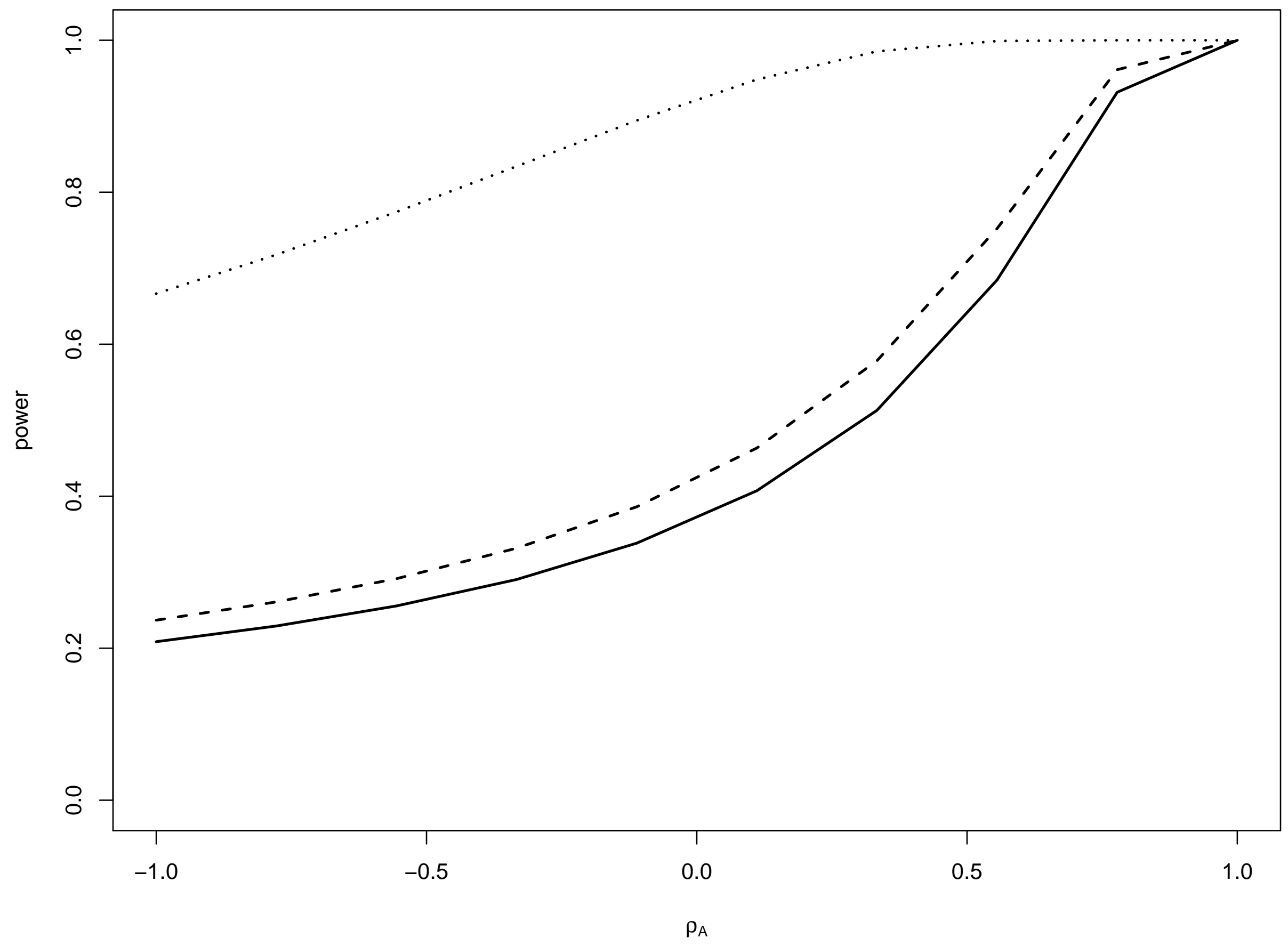




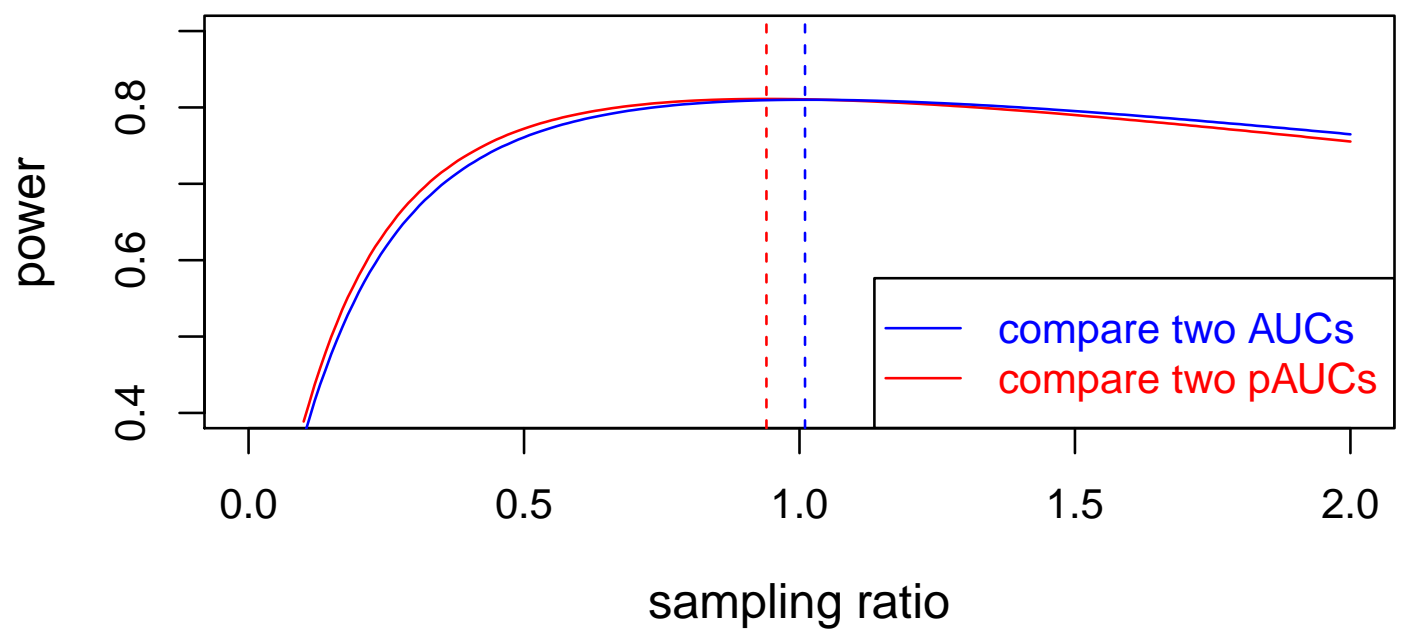




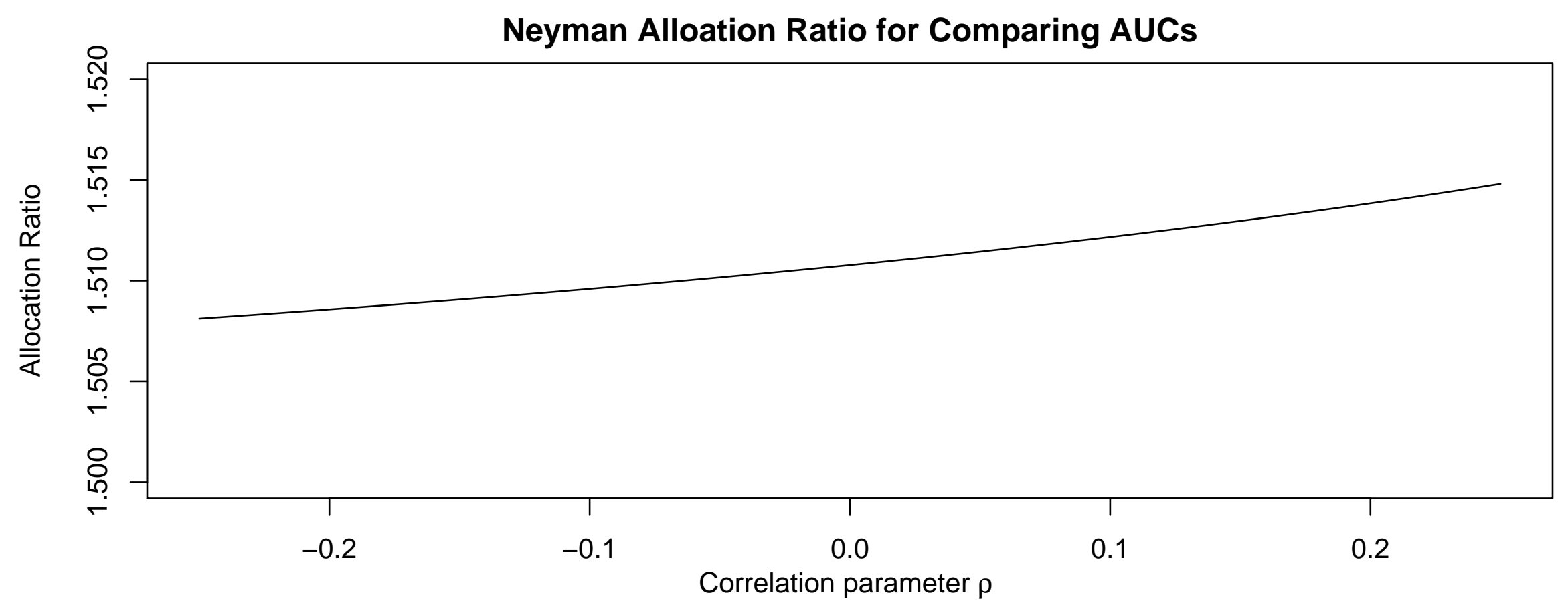

a) Bivariate Biexponential Variables

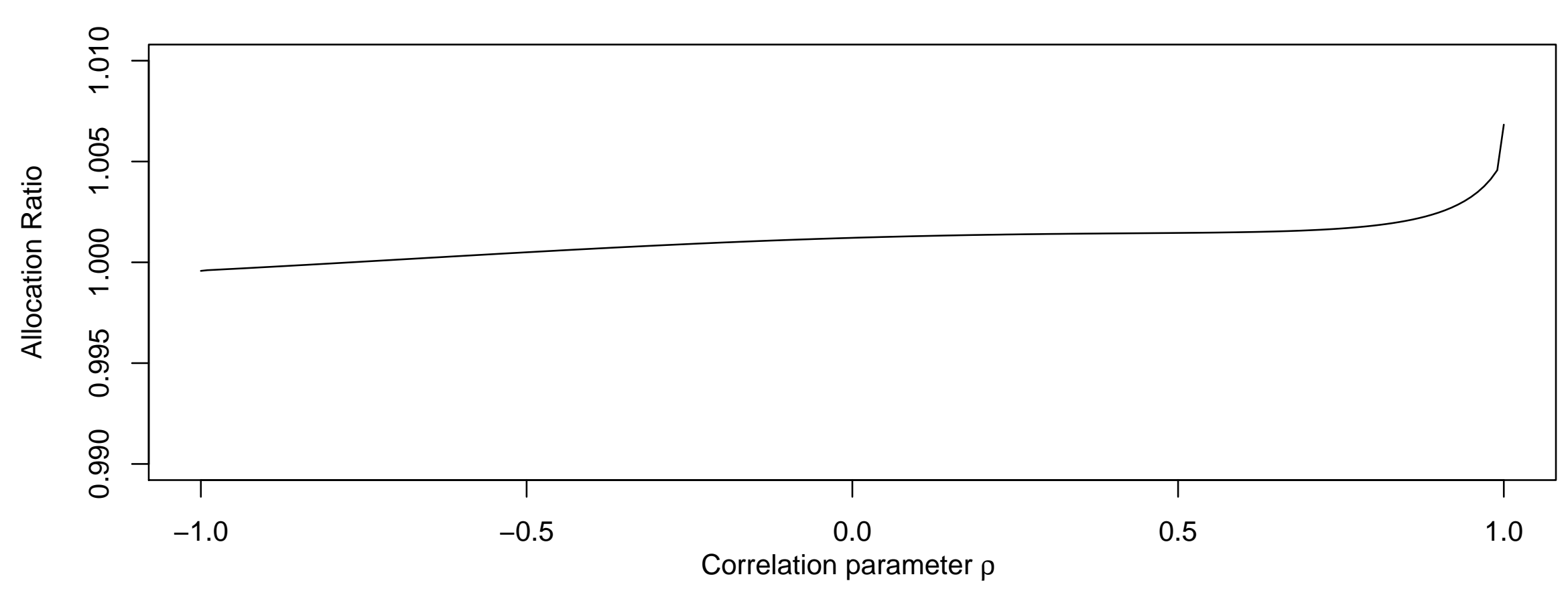

b) Bivariate Binormal Variables 